\title{
Gestão De Indicadores Farmacêuticos Aplicados À Farmácia Escola Em Joinville-Sc
}

\author{
Management Of Pharmaceutical Indicators Applied To Pharmacy School In Joinville-Sc \\ Gestión De Indicadores Farmacéuticos Aplicados A La Farmacia Escuela En Joinville-Sc
}

Deise Schmitz Bittencourt ${ }^{1}$, Graciele Schug Gonçalves ${ }^{2}$, Januaria Ramos Pereira Wiese ${ }^{3}$, Heidi Pfutzenreuter Carstens ${ }^{4}$.

Resumo: A Farmácia Escola é uma parceria entre ensino, serviço e comunidade, criada para desenvolver a profissão farmacêutica e atender os usuários do Sistema Único de Saúde na dispensação de medicamentos. O presente trabalho teve como objetivo analisar os indicadores farmacêuticos da Farmácia Escola, a fim de contabilizar os dados e identificar melhorias ou alterações para promoção da qualidade do serviço prestado. Foi realizada uma pesquisa quali-quantitativa retrospectiva do período de janeiro de 2014 a dezembro de 2018. Foram analisados os indicadores de 'atendimento da recepção', 'atendimentos por e-mail', 'total de atendimentos da dispensação' 'agendamentos

\footnotetext{
${ }^{1}$ Especialista em Gestão em Saúde. Farmacêutica Universidade da Região de Joinville. E-mail: schmitz.deise@gmail.com. https://orcid.org/0000-0002-9746$\underline{4287}$
}

${ }^{2}$ Especialista em Gestão em Saúde. Farmacêutica Universidade da Região de Joinville. E-mail: gracifar@gmail.com. https://orcid.org/0000-0002-1050-7353

\footnotetext{
${ }^{3}$ Mestre em Farmácia. Professora Adjunta - Universidade da Região de Joinville. E-mail: januariaramos@gmail.com. https://orcid.org/0000-0003-4053-6224
}

${ }^{4}$ Mestre em Saúde e Meio Ambiente. Professora Adjunta Farmacêutica - Universidade da Região de Joinville. E-mail: heidipi@hotmail.com. https://orcid.org/0000-0001-7082$\underline{0096}$ para dispensação', 'usuários que faltaram no dia agendado', 'atendimento de usuários faltantes (encaixes)', 'total de atendimentos da dispensação', 'total de atendimentos da FAE', e 'pacientes ativos CEAF'. O número total de atendimentos realizados na FAE, entre 2014 e 2018, foi de 745.406, com média mensal de 12.423. O absenteísmo foi observado na proporção de $13,1 \%$, mas houve um aumento importante na modalidade de atendimentos realizados por e-mail, demostrando que esta é uma ferramenta crescente no atendimento do usuário. Os resultados mostraram que os indicadores são excelentes ferramentas de gerenciamento e que é necessária uma avaliação continuada para melhoria dos processos e da gestão da FAE.

Palavras-chave: Farmácia, Administração de Serviços de Saúde; Serviços Comunitários de Farmácia

\footnotetext{
Abstract The Pharmacy School (Farmácia Escola - FAE) is a partnership between teaching, service and community created to
} 
develop the pharmaceutical profession and serve the users of the Brasilian Unified Health System in the dispensing of medicines. The present work aimed to analyze the pharmaceutical indicators from Pharmacy School, in order to account for the data and identify improvements or changes to promote the quality of the service provided. A qualitative and quantitative retrospective study was carried out from January 2014 to December 2018. The indicators of 'reception attendance', 'e-mail attendance', 'total dispensing attendance', 'dispensing schedules' , 'missed users', 'missing patient care', 'total dispensing care', 'total FAE care', and 'CEAF active patients'. The total number of visits performed at the FAE between 2014 and 2018 was 745,406 , with a monthly average of 12,423 . Absenteeism was observed in the proportion of $13.1 \%$, but there was a significant increase in the mode of attendance performed by e-mail, proving that this is an increasing tool in the user's service. The results showed that the indicators are excellent management tools and that a continuous evaluation is necessary to improve the processes and management of the FAE.

Key Words: Pharmacy; Health Services Administration; Community Phamacy Services

Resumen: La Farmacia Escuela (Farmácia Escola - FAE) es una asociación entre enseñanza, servicio y comunidad creada para desarrollar la profesión farmacéutica y atender a los usuarios del Sistema Único de Salud Brasileño en la dispensación de medicamentos. El presente trabajo tuvo como objetivo analizar los indicadores farmacéuticos de la Farmacia Escuela, a fin de contabilizar los datos e identificar mejoras o alteraciones para promover la calidad del servicio prestado. Se realizó una encuesta cuali-cuantitativa retrospectiva del período de enero de 2014 a diciembre de 2018. Se analizaron los indicadores de 'atención de la recepción', 'atendimientos por e-mail', 'total de atenciones de la dispensación' 'programaciones para dispensación' "usuarios que faltaron el día programado", "atención de usuarios faltantes (encajes) ',' total de atenciones de la dispensación ',' total de atenciones de la FAE ', y' pacientes activos CEAF '. El número total de atendimientos realizados en la FAE entre 2014 y 2018 fue de 745.406, con promedio mensual de 12.423. El absentismo fue observado en la proporción del $13,1 \%$, pero hubo un aumento importante en la modalidad de atendidas realizadas por email, demostrando que esta es una herramienta creciente en la atención del usuario. Los resultados mostraron que los indicadores son excelentes herramientas de gestión y que es necesaria una evaluación continuada para mejorar los procesos y la gestión de la FAE.

Palabras -clave: Farmacia; Administración de los Serviços de Salud; Servicios Comunitarios de Farmacia; 


\section{Introdução}

A Farmácia Escola SUS/SMS/Univille (FAE) foi criada para desenvolver a profissão farmacêutica e atender os usuários do Sistema Único de Saúde (SUS), funcionando mediante uma parceria firmada entre a Prefeitura Municipal de Joinville e a Universidade da Região de Joinville - Univille. São dispensados 208 diferentes tipos de medicamentos do Componente Especializado da Assistência Farmacêutica (CEAF), medicamentos que foram obtidos por meio de ações judiciais do Município de Joinville (Não Padronizados), medicamentos específicos controlados do Município (Morfina e Metadona) e medicamentos específicos do componente básico do Município (Cilostazol e Óleo Mineral).

A solicitação e dispensação de medicamentos do CEAF é uma estratégia para o acesso a medicamentos de alto custo fornecidos pelo SUS, em nível ambulatorial, mantendo a integralidade do serviço. Para o paciente ter o acesso e dar continuidade ao tratamento deve atender às linhas de cuidado que estão definidas em Protocolos Clínicos e Diretrizes Terapêuticas (PCDT), os quais são analisados pela Diretoria de Assistência Farmacêutica (DIAF) estadual e publicados pela Comissão Nacional de Incorporação de Tecnologias no SUS (CONITEC) do Ministério da Saúde.

Para retirar o medicamento na FAE, o usuário apresenta o receituário médico com o Código Internacional da Doença (CID) e o medicamento prescrito. Se o medicamento for disponível pelo PDCT, é entregue um checklist com as informações que o mesmo necessita para solicitar o medicamento e os respectivos formulários de preenchimento médico, conforme a patologia. É enviada a solicitação para autorização junto à DIAF que irá avalia-la. Se autorizadas, os usuários são contatados e agendada uma data e horário para o mesmo retirar o medicamento na FAE mensalmente.

Para qualificar e gerir um serviço como o descrito, devem ser criados mecanismos que possam quantificá-lo, detalhá-lo e analisá-lo, além de avaliar se as metas estabelecidas estão sendo atingidas ou precisam ser revistas. Ao visar o acompanhamento e avaliação continuada das ações, faz-se necessária a implantação de indicadores da assistência farmacêutica, que irão auxiliar o gerenciamento adequado do trabalho desenvolvido, qualificar o atendimento ao usuário, promovendo a racionalização dos recursos, ou quando necessário, a solicitação de $\operatorname{recursos}^{(1)}$. A criação de indicadores é importante para a avaliação da qualidade, pois proporciona uma medida e permite o monitoramento e a identificação de oportunidades de melhoria de serviços e de mudanças para o alcance da qualidade $^{(2)}$. No sistema de saúde, é difícil gerar uma equidade nos indicadores, devido a sua variabilidade dependendo do serviço ${ }^{(3)}$.

Por meio da análise de indicadores, é possível identificar fragilidades e prover 
informações confiáveis quanto ao serviço, constituindo uma ferramenta importante para verificação do alcance dos objetivos propostos ${ }^{(4)}$. Os indicadores podem ser empregados para analisar se as suposições que estão por trás da estratégia são válidas ou não, ou seja, analisar se as metas e objetivos estão sendo alcançados com a estruturação dos indicadores propostos ${ }^{(5)}$.

O farmacêutico possui como importante atribuição a definição, avaliação e monitoramento de indicadores e deve elaborálos como instrumento que operacionaliza as intenções expressas no seu âmbito de trabalho, com detalhamento das ações e metas, para ter uma avaliação concreta. Os indicadores expressam o acesso e a qualidade da organização do sistema de saúde, propagando características locais e da organização do sistema, podendo sofrer ajustes de acordo com a necessidade local. Também é importante ressaltar que os indicadores da assistência farmacêutica são específicos e devem ser pactuados conforme necessidade local, que contemple ações necessárias ao fortalecimento do local de saúde ${ }^{(6)}$.

Na FAE, todo o atendimento prestado ao usuário é contabilizado por meio de indicadores farmacêuticos, mas apesar de tais indicadores serem contabilizados, os mesmos não eram adequadamente analisados. Diante do exposto, o objetivo deste trabalho foi avaliar os indicadores de atendimento e dispensação da FAE, a fim de propor melhorias no processo de atendimento ao usuário e manejo dos problemas, promovendo qualidade nos serviços prestados ao usuário a partir da gestão.

\section{Revisão De Literatura}

O Sistema Único de Saúde (SUS) regulamenta e promove a seleção, programação, aquisição, armazenamento, distribuição e dispensação dos medicamentos do CEAF e os usuários podem ser advindos tanto do atendimento SUS quanto do particular $^{7}$. Desta forma, o CEAF atende todo paciente que possuir sua doença comprovada e atender os critérios de inclusão dos protocolos clínicos e diretrizes terapêuticas (PCDT), praticando o princípio da equidade, em que iguala o acesso da população aos medicamentos de alto custo ${ }^{8}$.

É mediante a parceria entre a União, o estado de Santa Catarina e o município de Joinville, que a Farmácia Escola SUS/SMS/Univille (FAE) dispensa para a população de Joinville os medicamentos do CEAF. Na Farmácia Escola são atendidos os usuários desde a solicitação até a dispensação de medicamentos, além do monitoramento dos pacientes, promovendo a Assistência Farmacêutica.

\section{A Assistência Farmacêutica é o} conjunto de ações e de serviços, que visam assegurar a assistência terapêutica integral e a promoção, proteção e recuperação da saúde nos estabelecimentos públicos e privados que desempenham atividades farmacêuticas. É 
uma perspectiva de conduta do farmacêutico perante o usuário do medicamento, em que o profissional estabelece uma relação estreita e acolhedora com o mesmo, comprometendo-se com o sucesso de sua farmacoterapia ${ }^{9}$. $\mathrm{Na}$ FAE, procura-se estimular a Assistência Farmacêutica, auxiliando na formação profissional do acadêmico de farmácia e acolhendo os usuários que frequentam o serviço, promovendo o estímulo farmacêutico sólido da universidade e apoio da prefeitura para que as ações de Assistência Farmacêutica possam ser promovidas concretamente.

Para realizar um melhor atendimento na FAE, faz-se necessária a implantação de indicadores da assistência farmacêutica, que auxiliam o gerenciamento adequado do trabalho desenvolvido, qualificam o atendimento ao usuário, promovem a racionalização dos recursos, ou quando necessário, a solicitação de recursos. Para isso, os resultados devem ser analisados e discutidos para serem adaptados ao contexto, estabelecendo utilidade, descrição, padrão, fontes, métodos e periodicidade de coleta dos dados e cálculos ${ }^{1}$.

O indicador pode ser definido como um sensor que auxilia na verificação se os objetivos propostos foram ou não alcançados. Este processo é algo positivo, uma vez que estabelece objetivos mais próximos ao ideal ${ }^{2}$. No que diz respeito à formulação de indicadores, torna-se primordial contextualizar o desempenho dentro do contexto estratégico, o que definirá os indicadores apropriados para a estratégia almejada $^{10}$. Para a conformação dos indicadores, são condicionantes o seu funcionamento e os dados que se espera buscar, gerando uma análise da avaliação do desempenho, conforme aquilo que é desejado dentro do comportamento do indicador ou pode ser alterado para que se tenha um dado em que se possa trabalhar ${ }^{3}$.

A criação de indicadores é importante para a avaliação da qualidade, pois proporciona uma medida e permite $\mathrm{o}$ monitoramento e a identificação de oportunidades de melhoria de serviços e de mudanças para o alcance da qualidade ${ }^{2}$.

Os indicadores vêm sendo usados como instrumento de apoio às decisões na elaboração do desenvolvimento do trabalho e devem simplificar a informação de fenômenos complexos, além de melhorar a comunicação entre o decisor e o processo ${ }^{11}$. Os indicadores podem ser empregados para analisar se as suposições que estão por trás da estratégia são válidas ou não, ou seja, analisar se as metas e objetivos estão sendo alcançados com a estruturação dos indicadores propostos ${ }^{5}$.

Segundo Manzini et. al. ${ }^{6}$ o farmacêutico deve realizar uma permanente avaliação da assistência farmacêutica por meio de indicadores específicos, que possibilitem o aprimoramento e gerenciamento do local de saúde. Os indicadores expressam o acesso e a qualidade da organização do sistema de saúde, propagando características locais e da 
organização do sistema, podendo sofrer ajustes de acordo com a necessidade local. Também é importante ressaltar que os indicadores da assistência farmacêutica são específicos e devem ser pactuados conforme necessidade local, que contemplem ações necessárias ao fortalecimento do local de saúde.

Nas farmácias, a utilização de indicadores é uma das maneiras mais confiáveis e fáceis de monitorar o desempenho da gestão, isto se dá na deliberação e análise dos resultados, gerenciamento dos processos, buscando ações para evitar o erro, desperdício ou melhorar a rotina do colaborador ${ }^{12}$. Por consequência, os gestores dos serviços de saúde devem criar indicadores a partir de critérios de relevância, confiabilidade, capacidade de discriminação e validade, o que irá promover o monitoramento das dimensões do desempenho do sistema de saúde ${ }^{3}$.

\section{Método}

Foi realizada uma pesquisa qualiquantitativa retrospectiva por meio de levantamento dos indicadores coletados nos atendimentos da FAE do período de janeiro de 2014 a dezembro de 2018. Os indicadores farmacêuticos analisados haviam sido criados a partir da observação da necessidade e demanda local pelo gestor. Foram avaliados os seguintes indicadores e referidas descrições:
- Atendimento da recepção: são contabilizados os tipos de serviços prestados na recepção (informações prestadas, renovações realizada, entrega de medicamentos, agendamentos, captação de receita, entrega de check-list e atendimento dos medicamentos que estavam em falta);

- Atendimentos por e-mail: são contabilizadas as respostas aos usuários que solicitaram algum serviço prestado na FAE por meio de e-mail;

- Total de atendimentos da dispensação: são somados diariamente os atendimentos do CEAF e Não Padronizados, separadamente;

- Agendamentos para dispensação: são contabilizados por meio de relatório gerado do sistema Micromed ${ }^{\circledR}$ (Sistema de Agendamento de Usuários) os usuários com horário agendado no dia corrente, para retirada de medicamento aprovado. Em média são agendados, aproximadamente, cinco usuários a cada sete minutos, durante $\mathrm{o}$ horário de funcionamento da FAE, das $8 \mathrm{~h}$ às $17 \mathrm{~h}$, com um intervalo entre $13 \mathrm{~h} 30 \mathrm{~min}$ até $14 \mathrm{~h}$;

- Usuários que faltaram no dia agendado: são contabilizados por meio de relatório gerado do sistema Micromed ${ }^{\circledR}$ os usuários que faltaram no horário pré-agendado;

- Atendimento de usuários faltantes (encaixes): são contabilizados os encaixes dos usuários que faltaram no dia agendado 
ou daqueles que necessitaram antecipar o atendimento;

- Total de atendimentos da dispensação: são somados diariamente os atendimentos do CEAF e Não Padronizados, separadamente;

- Total de atendimentos da FAE: são somados diariamente os atendimentos do CEAF, atendimentos do Não Padronizados, atendimento psicotrópico do elenco básico, atendimento dos medicamentos do elenco básico, atendimentos da recepção, atendimentos para solicitação de medicamentos do CEAF, atendimento por e-mail e acolhimento aos usuários;

- Pacientes ativos CEAF: dado obtido por meio do sistema Sismedex ${ }^{\circledR}$ por relatório gerencial.

Esclarece-se que foram incluídos os indicadores que estavam ativos, ou seja, aqueles que já haviam sido contabilizados previamente no período do estudo e excluídos os indicadores que estavam inativos ou incompletos. Os dados foram analisados por cálculo percentual e organizados em gráficos e tabelas no sistema Microsoft Excel ${ }^{\circledR}$ com consequente desvelamento das informações.

\section{Resultados E Discussão}

Apenas contabilizar números não é suficiente para o serviço de saúde, é necessário gerenciar os indicadores ${ }^{(1)}$. Sendo assim, os resultados apresentam os indicadores quantificados e a utilidade de cada um dentro do processo de gestão da Farmácia Escola.

$\mathrm{O}$ indicador de contagem diária permitiu a avaliação dos atendimentos totais realizados na FAE, entre os anos de 2014 e 2018. Foram somados todos os tipos de atendimentos de dispensação de medicamentos (CEAF e Não Padronizado, psicotrópicos e medicamentos do elenco básico), atendimentos na recepção, solicitações de medicamentos do CEAF, atendimentos por e-mail e acolhimentos. O total de atendimentos em 5 anos foi de 745.406 com média de 12.423 paciente/mês (Gráfico1).

\section{Gráfico 1 - Contagem diária com atendimento total da FAE.}




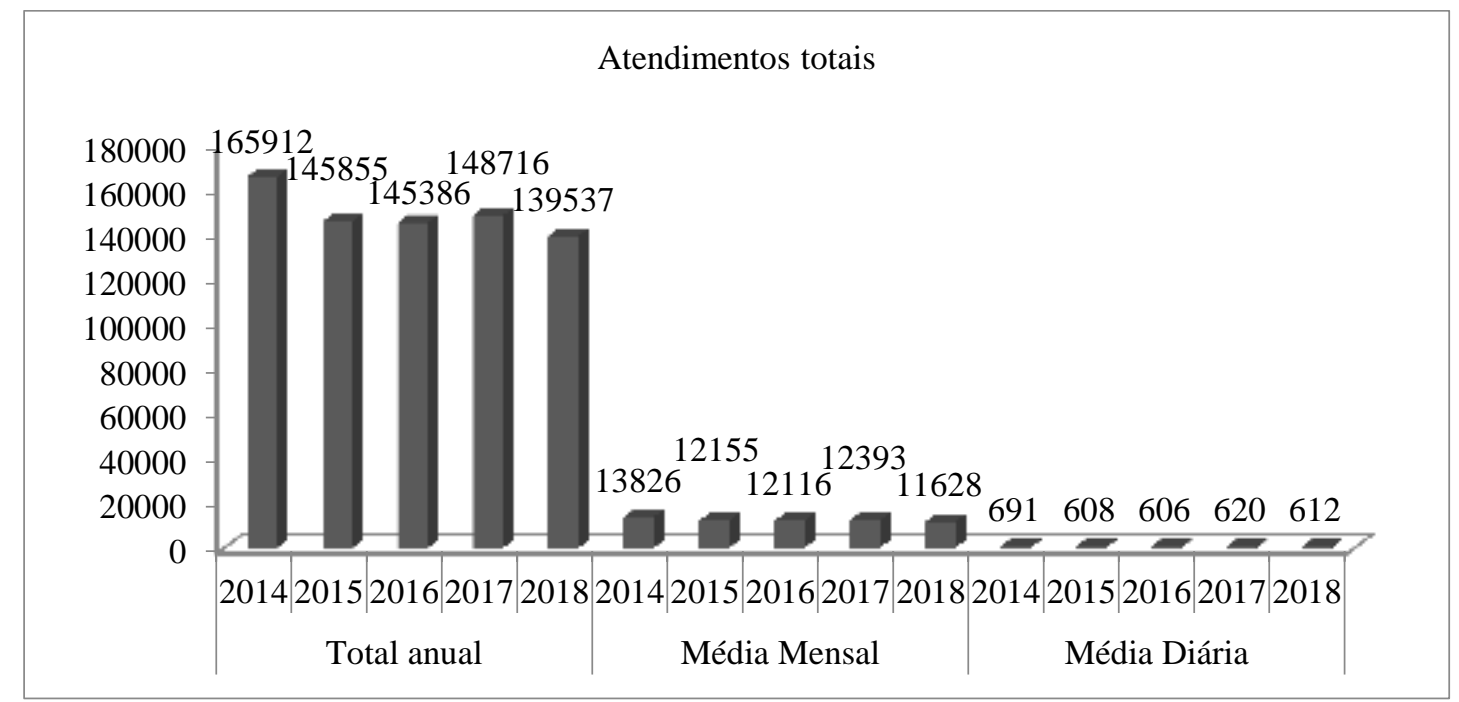

Fonte: Primária (2019)

Houve uma redução de atendimentos de 2014 para os demais anos, devido à retirada da dispensação do medicamento diosminathesperidina e mais uma redução de 2017 para 2018 pelo surgimento da possibilidade das dispensações do CEAF ocorrerem bimestralmente. Essas modificações, que resultaram em diminuição do número de pacientes, foram importantes para manutenção do fluxo de trabalho, considerando que o aumento do espaço físico e de funcionários é bastante limitado.

Prevenir, promover e principalmente recuperar a saúde do usuário que adentra ao serviço de saúde é desafiador, pois é necessário mantê-lo de forma integral com a escuta qualificada do usuário no serviço de saúde, mesmo com expressiva demanda (1,713). No presente estudo, os indicadores de contagem diária da FAE buscaram priorizar a geração de resolubilidade, adequando o número de atendimentos ao serviço, além de torná-lo mais humanizado.

Ainda que o número total de atendimentos tenha diminuído, foi observado que houve contínuo crescimento dos pacientes ativos do CEAF. A taxa de aumento foi de $27 \%$ entre 2014 e 2018, ou seja, 1.733 pacientes a mais para serem atendidos mensalmente. A pactuação da FAE com o almoxarifado do estado para envio bimestral (ao invés de mensal) foi a estratégia utilizada para tentar reduzir o número de atendimentos/dia com o volume crescente de usuários adentrando ao serviço.

$\mathrm{O}$ indicador de contagem total diária na dispensação corresponde à somatória dos usuários atendidos na dispensação, compreendendo CEAF e Não Padronizado. Foram atendidos, nos 5 anos de estudo, 410.294 usuários na dispensação (Gráfico 2). 


\section{Gráfico 2 - Indicador de contagem total diária na dispensação.}

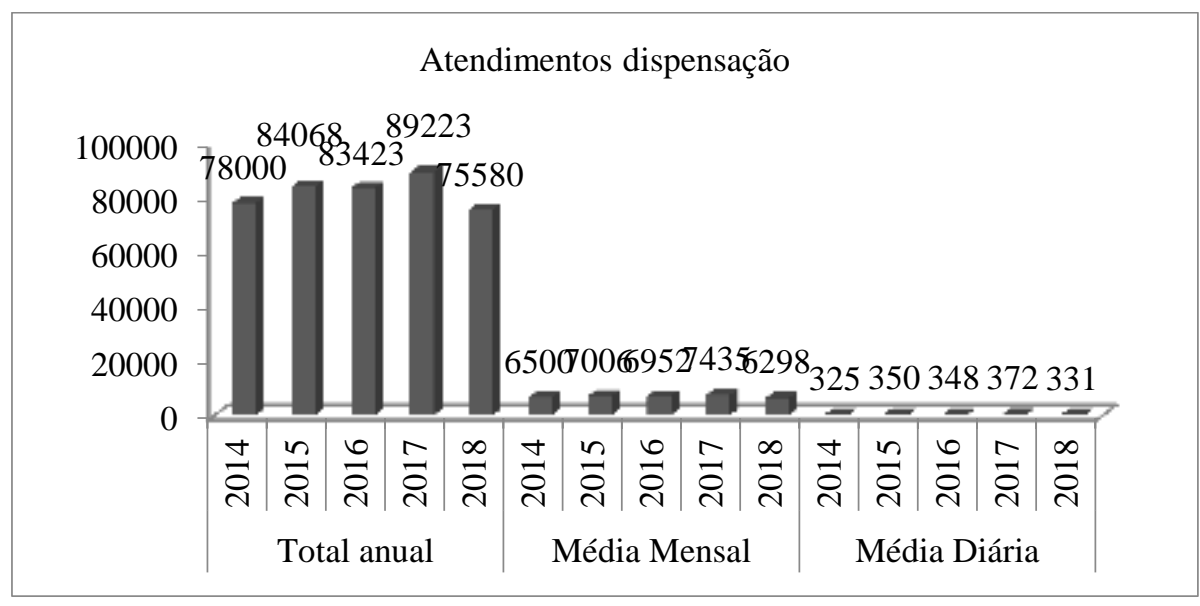

Fonte: Primária (2019)

Com base na análise do Gráfico 3, verificou-se 75.997 dispensações de medicamentos Não-Padronizados, com um decréscimo de $36 \%$ de pacientes entre os anos estudados. Tal ocorrência foi motivada por alguns acontecimentos como a migração de classes de medicamentos para o CEAF, a criação de protocolos municipais para a solicitação de medicamentos advindos por ação judicial extensiva, a revogação de ações judiciais extensivas e a redução da judicialização de medicamentos pela criação de câmaras técnicas municipais que auxiliam juízes na formulação da sentença.

Gráfico 3 - Contagem diária dos atendimentos do Não Padronizado.

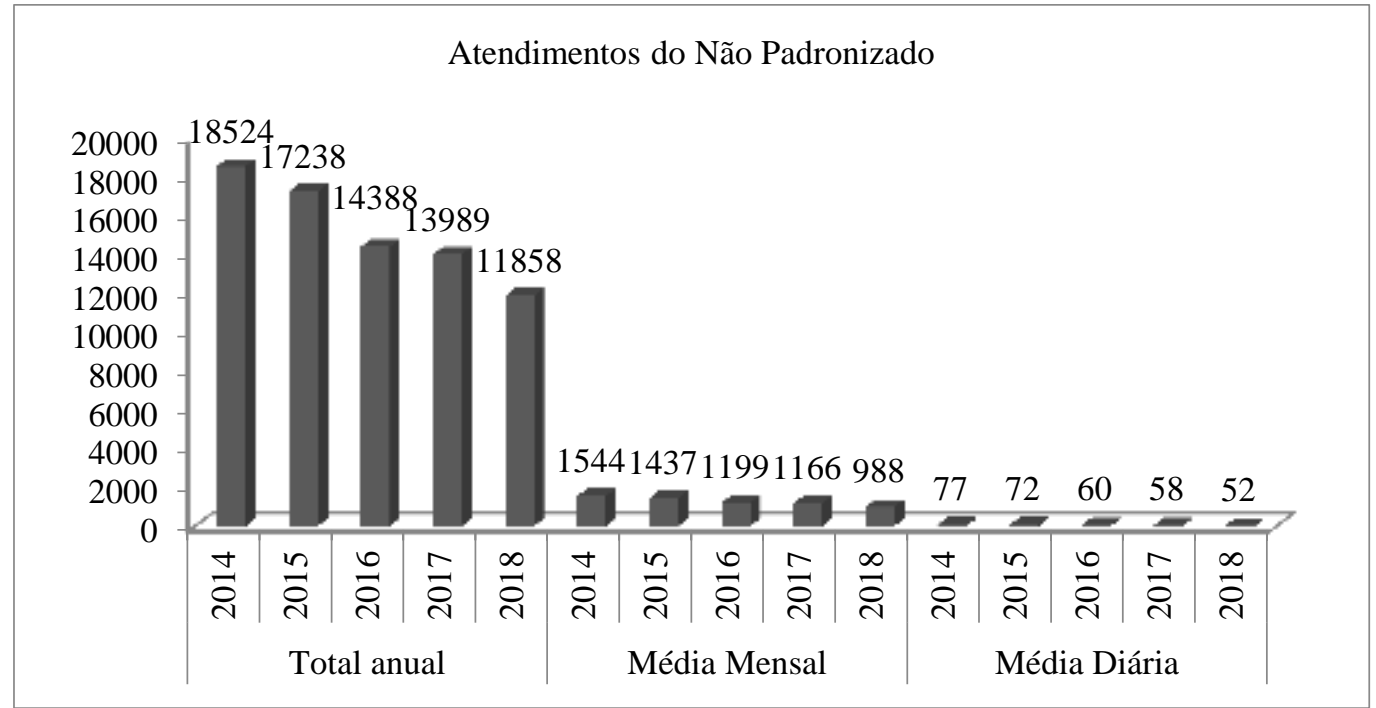

Fonte: Primária (2019) 
No Brasil, a grande judicialização da saúde, levou à busca de pareceres que não fossem decisões "solitárias" do juiz, mas sim coordenadas e estratégicas ${ }^{(14)}$. No município de Joinville, houve a criação do Núcleo de Apoio Técnico (NAT-Jus), que auxilia na decisão judicial $^{(15)}$, além da criação de Comitês Municipais de Saúde que fornecem recomendações aos juízes podendo decidir as demandas, que lhes são apresentadas. Dessa forma, houve uma redução da judicialização da saúde de 2014 até 2018.

As dispensações na Farmácia Escola são realizadas com agendamento prévio de data e horário. Foram realizados 384.972 agendamentos entre 2014 e 2018, o que equivale a uma média de 6.416 usuários agendados por mês. $\mathrm{O}$ absenteísmo foi observado na proporção de $13,1 \%$ ao mês, não havendo diferenças significativas entre os anos. Outro indicador analisado foi o número de encaixes de pacientes que faltaram em seu agendamento. Observou-se que a somatória dos anos de 2015 a 2018, foi de 21.755 usuários, com média mensal de 472 pacientes. Uma análise pertinente é a comparação entre os pacientes que faltaram no dia agendado e o número de encaixes de pacientes faltantes por mês. Considerando que a média de faltas é de 840 pacientes/mês, observa-se que $56 \%$ dos que faltaram no horário agendado do mês não retornam em outro dia para a retirada do medicamento, ou seja, não realizarão o tratamento no referido mês. Ressalta-se, que o custo de doença não controlada é maior e isso ocorre principalmente porque os pacientes procuram mais a emergência e são hospitalizados com maior frequência ${ }^{(16)}$.

O indicador da recepção teve seu início em setembro de 2015 e seus resultados estão apresentados na tabela 1.

Tabela 1 - Atendimentos da recepção.

\begin{tabular}{ccccc}
\hline Detalhamento & $\mathbf{2 0 1 5 *}$ & $\mathbf{2 0 1 6}$ & $\mathbf{2 0 1 7}$ & $\mathbf{2 0 1 8}$ \\
\hline Informação & 2776 & 9430 & 8163 & 8414 \\
Renovação & 1618 & 5838 & 6131 & 7870 \\
Entrega de & 937 & 4210 & 4891 & 5831 \\
Medicamento & 1432 & 5276 & 6476 & 6099 \\
Agendamento & 42 & 219 & 256 & 310 \\
Captação & & & & \\
\end{tabular}




\begin{tabular}{ccccc}
\hline $\begin{array}{c}\text { Entrega de check-list } \\
\text { Entrega de medicação } \\
\text { em falta }\end{array}$ & 545 & 2206 & 2265 & 2331 \\
& 0 & 1904 & 5006 & 1911 \\
\hline
\end{tabular}

\section{*Dados a partir de setembro de 2015}

Fonte: Primária (2019)

Pode-se observar que, apesar da recepção realizar um atendimento de livre demanda e, por esse motivo, difícil de prevê-lo em números, os anos de 2016, 2017 e 2018 possuem valores similares no quesito 'entrega de checklist', 'captação' e 'agendamento'. A 'renovação' teve um acréscimo de $25,8 \%$ e 'entrega de medicamentos' de $27,8 \%$, o que é reflexo do aumento do número de pacientes ativos no CEAF, já discutido anteriormente. O decréscimo observado no item 'informação', pode estar relacionado à nova modalidade de atendimento incorporada ao serviço da FAE. $\mathrm{O}$ atendimento por e-mail foi uma maneira específica pensada para melhor comodidade do paciente, que é utilizada para retirar dúvidas dos usuários, saber informações sobre a falta de medicamentos, informações sobre autorizações de novos pedidos de medicamentos, agendar solicitação de medicamento ou reagendar horário de atendimento. Este indicador foi iniciado em fevereiro de 2018, juntamente com o serviço, e atingiu 5.627 usuários, contabilizando em média 27 pacientes/dia, os quais não precisam se deslocar até a Farmácia Escola para solicitar informações. $\mathrm{O}$ atendimento por email não é realizado por uma pessoa específica, sendo feito pelo servidor público que possuir um tempo livre entre um atendimento e outro ou no final do dia, porém é mais viável atualmente do que o atendimento telefônico, pois pode ser feito de maneira flexível.

A recepção é o primeiro local do serviço de saúde que o usuário está em contato e um atendimento humanizado poderá gerar a aproximação e inclusão do paciente, promovendo uma escuta qualificada das necessidades dos usuários ${ }^{(13)}$. Dessa maneira, a análise dos seus indicadores permitiu uma visão dos tipos de atendimentos realizados ao longo do tempo e levou à adequação dos servidores para prestação dos serviços de forma humanizada, verificando as necessidades dos pacientes com resolução da melhor forma possível dos seus problemas.

\section{Conclusão}

A FAE promoveu a implantação e análise dos indicadores farmacêuticos, os quais foram utilizados para administrar o 
serviço e propor melhorias. $\mathrm{O}$ atendimento aos usuários envolve principalmente os acadêmicos de farmácia e tem como objetivo principal ensiná-los a profissão farmacêutica. A partir da apresentação dos indicadores e de suas aplicabilidades, são fortalecidas as habilidades de gerenciamento farmacêutico, mostrando a importância dos indicadores na administração dos serviços de saúde.

O indicador é um sensor que contribui para a verificação dos objetivos propostos, devendo ser contextualizados de forma estratégica e alterados quando necessário. Por meio dos indicadores analisados na Farmácia Escola, maximizou-se poder de decisão e análise dos resultados e foram identificadas fragilidades e geradas informações confiáveis quanto ao serviço.

\section{Referências}

1. Brasil. Conselho Nacional de Secretários de Saúde. Assistência de Média e Alta Complexidade no SUS. Conselho Nacional de Secretários de Saúde. Brasília: CONASS, 2007.

2. D’Innocenzo, M.; Adami, N. P.; Cunha, I. C. K. O. O movimento pela qualidade nos serviços de saúde e enfermagem. Rev Bras Enferm, vol. 59, n. 1, p. 84-8, janfev, 2006.

3. Viacava, F.; Ugá, M. A. D.; Porto, S.; Laguarda, J.; Moreira, R. S. Avaliação de Desempenho de Sistemas de Saúde: um modelo de análise. Ciência \& Saúde Coletiva, vol. 17, n. 4, p. 921-934, 2012.

4. Rover, M. R. M.; Vargas-Pelaéz, C. M.; Farias, M. R.; Leite, S. N. Metodologia para o desenvolvimento de um protocolo de indicadores para a avaliação da capacidade de gestão da assistência farmacêutica. Departamento de Ciências Farmacêuticas, Universidade Federal de Santa Catarina. Florianópolis, SC. Brasil, 2016.

5. Kiyan, F. M. Proposta para desenvolvimento de indicadores de desempenho como suporte estratégico. Tese de Doutorado. Universidade de São Paulo, 2001.

6. Manzini, F. et al. O farmacêutico na assistência farmacêutica do SUS: diretrizes para ação. Conselho Federal de Farmácia - Brasília, 2015.

7. Marin, N.; Luiza, V. L.; Osorio-de-Castro, C. G. S.; Machado-dos-Santos, S. Assistência farmacêutica para gerentes municipais. Rio de Janeiro: OPAS/OMS, 2003.

8. Boing, A. C.; Bertoldi, A. D.; Boing, A. F.; Bastos, J. L.; Peres, K. G. Acesso a medicamentos no setor público: análise de usuários do Sistema Único de Saúde no Brasil. Cad. Saúde Pública, Rio de Janeiro, 29(4): p. 691-701, abr, 2013.

9. Oliveira, L. C. F.; Assis, M. M. A.; Barboni, A. R. Assistência farmacêutica 
no Sistema Único de Saúde: da Política Nacional de Medicamentos à Atenção Básica à Saúde. Ciência \& Saúde Coletiva, 15(Supl. 3):3561-3567, 2010.

10. Carneiro, J.; Silva, J. F.; Rocha, A., Hemais, C. A. Mensuração do desempenho organizacional: questões conceituais e metodológicas. Estudos em negócios IV, p. 145-175, 2005.

11. Ventura, S. K.; Reis, L. F. R.; Takayanagui, A. M. M. Avaliação do gerenciamento de resíduos de serviços de saúde por meio de indicadores de desempenho. Eng Sanit Ambient, vol.15, n. 2, p. 167-176, abr/jun, 2010.

12. Ferreira, C. A. A.; Nunes, G. L. Z.; Souza, W. I.; Vianna, B. L. B.; Guimarães, H. A. A.; Azevedo, M. A. G. Monitoramento da gestão farmacêutica com o uso de Indicadores em um Hospital Público. Rev. Bras. Farm. Hosp. Serv. Saúde São Paulo vol. 4 n.2, p. 14-18, abr./jun. 2013.

13. Brasil. Ministério da Saúde. Acolhimento nas práticas de produção de saúde / Ministério da Saúde, Secretaria de Atenção à Saúde, Núcleo Técnico da Política Nacional de Humanização. - 2. ed. 5. reimp. - Brasília: Editora do Ministério da Saúde, 2010.

14. Asensi, F. D.; Pinheiro, R. Judicialização da saúde no Brasil: dados e experiência. Brasília: Conselho Nacional de Justiça, 2015.
15. Município de Joinville. Secretaria de Saúde, Conselho Municipal de Saúde, Decreto $\mathrm{n}^{0}$ 30.043, de 14 de novembro de 2017. Institui o Núcleo de Apoio Técnico ao Sistema de Justiça - NAT-Jus, regulamenta os procedimentos voltados à prevenção e resolução administrativa de litígios na saúde e dá outras providências. Diário Oficial Eletrônico do Município de Joinville, 2017.

16. Costa E, Caetano; R, Werneck GL, Bregman M, Araújo DV, Rufino R. Estimativa de custo da asma em tratamento ambulatorial: estudo com dados de mundo real. Rev Saude Publica. 2018;52:27

Participação dos autores:

Carstens HP trabalhou na coleta de dados, análise estatística e elaboração e redação final do texto.

Bitencourt DS trabalhou na concepção teórica, metodologia, elaboração, análise estatística e redação final do texto.

Gonçalves GS trabalhou na concepção teórica e elaboração dos resultados.

Wiese JRP trabalhou na concepção teórica e metodologia.

Recebido: 17.07.2019

Revisado: 12.08.2019

Aprovado: 13.09.2019 